\title{
IJMSR
}

\section{Riscos cardiovasculares em adolescentes praticantes de basquete}

\author{
Priscila Saiury Cassemiro Silva ${ }^{1}$ \\ Valdir Neto de Moura Azevedo ${ }^{2}$ \\ Patrícia Espíndola Mota Venâncio ${ }^{3}$
}

\begin{abstract}
RESUMO
Este estudo tem como objetivo identificar e correlacionar os riscos cardiovasculares, dentre eles hipertensão, obesidade e percentual de gordura, em praticantes de basquete pertencentes à Entidade Vultures de Anápolis. Este trabalho avaliou 39 atletas de basquetebol, divididos em quatro categorias (sub13, sub15, sub17 e sub19), de quem se aferiu a PA com cinco minutos em repouso, Circunferência de Cintura, Índice de Massa Corporal, Estatura e o Peso. Foi utilizado para melhor compreensão dos resultados o Test " $\mathrm{t}$ " de Student, Mann-Whitney U, Wilcoxon e ANOVA para a comparação dos

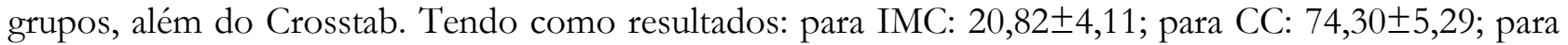

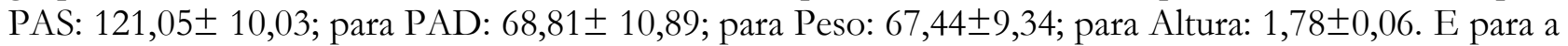
correlação das variáveis obteve-se: peso x estatura: 0,863; peso x CC:0,865; peso x IMC:0,776; estatura x CC: 0,618 ; CC x IMC:0,826**. Concluiu-se que a maioria dos praticantes de basquete não possui riscos cardiovasculares, e, ao correlacionar os riscos cardiovasculares, pode ser percebido que quanto mais alto e mais pesado o atleta, maiores foram os valores de Circunferência de Cintura e de Índice de Massa Corporal.
\end{abstract}

Palavras-chave: Risco cardiovascular; Basquetebol; Adolescente.

\section{AbStract}

This study aims to identify and correlate cardiovascular risks, including hypertension, obesity and fat percentage, in basketball players belonging to Entity Vultures of Anápolis. This study evaluated 39 basketball athletes, who were divided into four categories (sub13, sub15, sub17 and sub19), whose BP was measured with five minutes at rest, measurement of Waist Circumference, Body Mass Index, Height and Weight. Student's "t" test, Mann-Whitney U, Wilcoxon and Anova were used to better understand the results for the comparison of the groups, in addition to the Crosstab. The results: for BMI: $20.82 \pm 4.11$; for CC: $74.30 \pm 5.29$; for PAS: $121.05 \pm 10.03$; for PAD: $68.81 \pm 10.89$; for Weight: $67.44 \pm$ 9.34; for Height: $1.78 \pm 0.06$. And for the correlation of variables, we obtained: weight x height: 0.863; weight x CC: 0.865; weight x BMI: 0.776; height x WC: 0.618; WC x BMI: 0.826 **. It was concluded that the majority of basketball players do not have cardiovascular risks, and, when correlating cardiovascular risks, the higher and heavier the athlete, the higher the Waist Circumference and Body Mass Index values.

Keywords: Cardiovascular risk; Basketball; Teenager.

Submissão: $13 / 01 / 2021$

Aceite: 19/03/2021

\footnotetext{
${ }^{1}$ UniEvangelica, Educação Física, Anápolis

2 UniEvangelica, Educação Física, Anápolis

${ }^{3}$ Centro Universitário de Anápolis, Anápolis, GO. Coordenadora do curso de Educação Física.

venanciopatricia@hotmail.com
} 
As doenças cardiovasculares são as principais causas de morbidade e mortalidade em todo o mundo. Representando riscos para vários grupos de indivíduos, sendo consideradas um problema de longo prazo, afetando todas as faixas etárias ${ }^{1}$.

São também conhecidas como cardiopatias. Esse termo descreve de forma geral as doenças que afetam o coração e os vasos sanguíneos. Existem diferentes tipos de doenças cardiovasculares com diferentes causas e grau de agressividade, sendo uma delas o sobrepeso ${ }^{2}$.

Outras doenças cardiovasculares incluem insuficiência cardíaca congestiva, acidente vascular cerebral, defeitos cardiovasculares congênitos, hipertensão, endurecimento ou estreitamento (aterosclerose) dos vasos sanguíneos, incluindo as artérias coronárias e outras doenças do sistema circulatório, Diabetes, colesterol alto, obesidade, falta de atividade física, tabagismo, aumento da idade e histórico familiar são fatores de risco para doenças cardiovasculares. ${ }^{2,3 .}$

A hipertensão é a doença que mais representa risco para os pacientes, pelo fato que a pressão arterial elevada pode gerar alterações nas paredes dos vasos sanguíneos e, caso não seja tratada, pode resultar em enfarte do miocárdio ${ }^{4}$.

A Hipertensão Arterial Sistêmica (HAS), conceituada como síndrome, caracteriza-se pela presença de níveis tensionais elevados, associados as alterações metabólicas e hormonais, em que muitas vezes pode ocorrer também por alterações como obesidade e sedentarismo ${ }^{5}$

Pertencente ao agrupamento de fatores de risco cardiovascular, a obesidade está relacionada com o percentual de gordura. Sabe-se que pessoas com obesidade correm maior risco de desenvolver hipertensão, diabetes, dislipidemia e síndrome metabólica (SM) ${ }^{6,7}$.

Os agentes promotores da obesidade são hormonais, hereditários, ingestão excessiva de alimentos e os baixos níveis de atividade física. Desses, na maioria dos casos, fatores como estilo de vida (atividade física) representam a combinação mais efetiva para o controle de peso e o desequilíbrio, sendo a principal causa do crescente índice de sobrepeso observado nos brasileiros ${ }^{7}$.

Para não adquirir obesidade nem outras doenças cardiovasculares, a prática de atividade física é recomendada, uma vez que promove o aumento no consumo de oxigênio, no débito cardíaco, no volume sistólico, na pressão arterial sistólica e no condicionamento de força ${ }^{6,7 .}$

A realização de atividade física consiste no movimento corporal que é produzido pela musculatura que gera o gasto de energia maior do que o de repouso. E como um dos exemplos de práticas de atividade física tem-se a modalidade de basquete ${ }^{8 .}$

$\mathrm{O}$ basquete, por ser uma modalidade que gasta muita energia e por ser um esporte intenso, tem como principais capacidades condicionantes a resistência aeróbia e anaeróbia, a velocidade e a força, principalmente a força de explosão. A pessoa que pratica frequentemente melhora o seu condicionamento físico e a sua capacidade cardiorrespiratória ${ }^{7}$.

A doença cardiovascular é caracterizada pelo funcionamento anormal do coração ou dos vasos sanguíneos. A doença cardíaca é um termo geral para uma variedade de condições cardíacas e a forma mais comum dessa enfermidade cardíaca, envolve as artérias coronárias ${ }^{8}$.

As doenças cardiovasculares representam riscos para os pacientes e é a principal causa de morte. As pessoas com essa condição possuem alto risco e, além do mais, é considerado um problema de longo prazo, já que não existe cura ${ }^{1}$.

Os fatores de risco da hipertensão arterial são causados, na maioria, por histórico familiar, sedentarismo, obesidade, colesterol e triglicerídeos elevados; necessitando, como-prevenção, mudanças no estilo de vida, com redução do sal, do álcool, do cigarro, e com busca pela prática de exercício físico ${ }^{10 .}$

A hipertensão é uma doença crônica que atinge milhões de pessoas independentemente de idade, raça, sexo e padrão social. Na maioria das vezes é sintomática, podendo apresentar tonturas, dores de cabeça, rubor facial, sangramento pelo nariz, dispneia, agitação, vômito, visão borrada e fadiga $^{10 .}$

Existe uma ligação entre o consumo de oxigênio $\left(\mathrm{VO}_{2}\right)$ do corpo e a aptidão cardiorrespiratória, pois a entrega de oxigênio aos tecidos depende da função pulmonar e cardíaca. O vo2 máximo é a taxa 
em que o oxigênio pode ser usado pelo corpo durante um trabalho intenso; está relacionado diretamente com a capacidade do coração de entregar sangue aos músculos. $\mathrm{O} \mathrm{VO}_{2}$ é um bom índice de aptidão cardiorrespiratória e um bom preditor de capacidade de desempenho em eventos aeróbicos, o que pode ser realizado, por exemplo com o basquete ${ }^{8 .}$

Para avaliar os riscos cardiovasculares citados, utilizamos a medição da circunferência da cintura (CC) para a obesidade que é considerada a maneira mais simples. A CC tem mostrado ser um dos indicadores antropométricos mais precisos da gordura abdominal. Ela está intimamente correlacionada com a relação cintura/quadril (RCQ) ${ }^{11}$. Os locais de medidas de CC variam, mas normalmente é indicada a medição da CC no plano horizontal entre as costelas inferiores e a crista ilíaca ${ }^{6,7 .}$

O local de medição influencia a magnitude da circunferência da cintura e a prevalência de obesidade abdominal. É recomendado que a circunferência em mulheres seja inferior a $88 \mathrm{~cm}$ e em homens inferiores a $102 \mathrm{~cm}$. Valores superiores podem representar risco de doenças cardiovasculares ${ }^{7}$.

Para identificação da hipertensão, a pressão arterial (PA) deve estar dentro da classificação de HAS sustentada, isto é, apenas uma aferição não nos autoriza o diagnóstico, pois a PA varia durante as 24 horas do dia, sendo normalmente mais baixa nos períodos de sono (descenso noturno). Por isso deve ser aferido com cinco minutos em repouso. $\mathrm{O} \mathrm{VO}_{2 \text { Máx }}$ pode ser medido ou previsto por testes de aptidão. $\mathrm{O} \mathrm{VO}_{2 \text { Máx }}$ é um bom índice de aptidão cardiorrespiratória e um bom preditor de capacidade de desempenho em atividades aeróbicas, como o basquete ${ }^{7,8}$

O desempenho ideal no basquetebol é altamente complexo, pois requer uma combinação de habilidades técnicas e táticas e alto grau de aptidão física. As equipes de basquete seguem treinamentos exigentes, tornando importante avaliar a capacidade de cada jogador, que deve estar exposto como parte do planejamento de curto e longo prazo para a equipe. Os fatores que diferenciam os jogadores de basquete profissional dos amadores são determinados pela capacidade anaeróbia que estes possuem $^{4,7}$

A prática de basquete exige um processo de seleção e análise dos potenciais e habilidades dos jogadores, a fim de possibilitar a obtenção de melhor resultados durante a partida, sempre avaliando o desempenho dos atletas ${ }^{12}$ É recomendada qualquer indivíduo, mesmo aqueles que possuem limitações físicas, visto que pode evitar o desenvolvimento de doenças cardiovasculares ${ }^{13 .}$

Diante do exposto, a seguinte pergunta norteia a realização deste estudo: será que até mesmo os praticantes de basquete possuem algum risco cardiovascular? Com base nos dizeres acima, frente das demandas que o basquete promove e sobre a escassez de estudos relevantes sobre essa população, fazse necessário o desenvolvimento desse estudo. Neste sentindo o objetivo do estudo foi identificar e correlacionar o risco cardiovascular em atletas adolescentes praticantes de basquete.

\section{MATERIAIS E MÉTODOS}

Trata-se de um estudo transversal, quantitativo e descritivo com uma população composta por $100 \%$ dos atletas homens pertencentes a Entidade Desportiva Vultures Anápolis Basquete, com a faixa etária entre 13 a 20 anos. Os participantes da pesquisa foram divididos por categoria, sendo a categoria sub13 com 10 atletas; a categoria sub15 com 13 atletas; a categoria sub17 com 07 atletas; e a categoria sub19 com 09 atletas, totalizando 39 praticantes de basquete.

Foram incluídos na pesquisa praticantes da modalidade de basquete com um período mínimo de seis meses de treinamento e com $75 \%$ de frequência. Para os que eram menores de idade os pais/responsáveis que concordaram, assinaram o Termo de Consentimento Livre e Esclarecido (TCLE). Após os atletas entregarem o termo de consentimento assinado foram iniciadas as coletas.

Os testes foram realizados no Centro Universitário de Anápolis - UniEvangélica, em dias alternados da semana, no turno vespertino, a partir das 14 horas, durante o mês de agosto de 2019, na cidade de Anápolis-GO. E antes da realização dos testes foi feito uma anamnese com os atletas.

Para a composição corporal, foi utilizada a medida da massa corporal (kg) em uma balança eletrônica (Tech 05, China). Os atletas subiram na balança descalços ou de meias, distribuindo o peso entre as duas pernas, olhando para frente e com a coluna ereta. Para a estatura, foi usado um 
estadiômetro portátil da marca Wiso (Santa Catarina, Brasil), com precisão de $0,1 \mathrm{~cm}$. Durante as medidas, os praticantes permaneceram na posição bipodal, com os calcanhares, panturrilhas, nádegas, escápulas e cabeça, encostados na parede. A posição da cabeça ocorreu de acordo com o plano de Frankfurt, e a medida foi anotada no momento da inspiração.

O índice de Massa Corporal (IMC) foi calculado a partir da divisão da massa corporal (em quilos) pela estatura (em metros), elevada à segunda potência $\left(\mathrm{kg} / \mathrm{m}^{2}\right)$. O IMC foi classificado de acordo com Tabela de IMC-para-idade.

A circunferência de cintura foi tomada com uma fita métrica (Sanny). O atleta levantou a camisa de modo que a fita passasse no entorno do abdômen, abaixo da última costela, utilizando a classificação do risco de doença cardiovascular a partir da circunferência de cintura.

A pressão arterial foi aferida por meio de um esfigmomanômetro e estetoscópio (Omron, 6124). O atleta teve de sentar-se e com o braço apoiado com um ângulo de $90^{\circ}$, foi colocado o esfigmomanômetro na região do bíceps e foi aferida a pressão, de acordo com as orientações da Sociedade Brasileira de Cardiologia.

Para a análise dos dados, foi utilizado um teste de normalidade. Para melhor compreensão dos dados gerados na pesquisa estes foram apresentados em forma de percentual com o desvio padrão, mínimo e máximo com o emprego dos seguintes testes:

Test "t" de Student, Mann-Whitney U, Wilcoxon. Foi utilizado também ANOVA para a realização da comparação dos grupos e Crosstab para verificar a existência de alguma diferença entre os grupos por meio do Software SPSS 20.0 com nível de significância $\mathrm{p} \leq 0,05$.

\section{RESUltados}

A Tabela 01 mostra os resultados quanto a comparação das seguintes variáveis: Peso, Estatura, IMC, CC, PAS e PAD entre as categorias Sub13, Sub15, Sub17 e Sub19. Percebe-se que a categoria

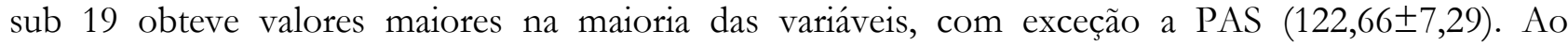
comparar os riscos cardiovasculares entre categorias, pressão arterial foi a única variável que não houve diferença significativa: PAS $\mathrm{p} \leq 0,325$ e para PAD $\mathrm{p} \leq 0,949$.

Tabela 1 - Comparação das variáveis entre as categorias

\begin{tabular}{cccccc}
\hline Variáveis & Sub13 & Sub15 & Sub17 & Sub19 & $\mathrm{p}$ \\
\hline Peso & $52,13 \pm 5,52$ & $59,48 \pm 7,37$ & $77,24 \pm 17,58$ & $80,94 \pm 6,90$ & 0,000 \\
Estatura & $1,61 \pm 0,06$ & $1,75 \pm 0,04$ & $1,86 \pm 0,08$ & $1,90 \pm 0,06$ & 0,000 \\
IMC & $19,90 \pm 1,98$ & $19,42 \pm 2,47$ & $21,70 \pm 2,54$ & $22,29 \pm 1,23$ & 0,013 \\
CC & $69,38 \pm 5,22$ & $70,66 \pm 4,68$ & $77,04 \pm 8,15$ & $80,14 \pm 3,14$ & 0,000 \\
PAS & $116,90 \pm 9,59$ & $119,07 \pm 11,59$ & $125,57 \pm 11,68$ & $122,66 \pm 7,29$ & 0,325 \\
PAD & $65,00 \pm 22,23$ & $69,38 \pm 7,76$ & $70,42 \pm 6,72$ & $70,44 \pm 6,85$ & 0,949 \\
& & & & & \\
\hline
\end{tabular}

$\mathrm{Na}$ Tabela 02 mostram-se os resultados das correlações entre os riscos cardiovasculares analisados, as principais relações encentradas foram: CC e IMC. (Peso x Estatura 0,863**); (Peso x CC 0,865**); (Peso x IMC 0,776**); (Estatura x CC 0,618**).

Tabela 2 - Correlações das variáveis

\begin{tabular}{ccc}
\hline Variaveis & $\mathrm{R}$ & $\mathrm{p}$ \\
Correlação & $0,863^{* *}$ & 0,000 \\
Peso x Altura & $0,865^{* *}$ & 0,000 \\
Peso x CC & $0,776^{* *}$ & 0,000 \\
Peso x IMC & $0,618^{* *}$ & 0,000 \\
Altura x CC & $0,826^{*}$ & 0,000 \\
CC x IMC &
\end{tabular}


A Tabela 3 mostra a classificação do IMC entre as categorias. A maioria dos avaliados está dentro da classificação normal. Porém as categorias Sub 19 e Sub 15 obtiveram valores de 88,88\% e $15,38 \%$ do percentual de sobrepeso, respectivamente.

Tabela 3 - Classificação de Índice de Massa Corporal

\begin{tabular}{lcccc}
\hline & Sub13 & Sub15 & Sub17 & Sub19 \\
IMC & $\mathrm{n}(\%)$ & $\mathrm{n}(\%)$ & $\mathrm{n}(\%)$ & $\mathrm{n}(\%)$ \\
Desnutrição aguda grave & -- & -- & -- & $1(11,11)$ \\
Desnutrição aguda moderada & $2(22,22)$ & -- & -- & -- \\
Normal & $7(77,77)$ & $11(84,61)$ & $7(100,00)$ & -- \\
Sobrepeso & -- & $2(15,38)$ & -- & $8(88,88)$ \\
\hline
\end{tabular}

A Tabela 4 refere-se à classificação da Pressão Arterial (PA) entre as categorias em que todas elas apresentaram a maioria dos participantes entre as classificações ótima e normal, não havendo diferença significativa entre categorias.

Tabela 4 - Classificação de Pressão Arterial

\begin{tabular}{lcccc}
\hline PA & Sub13 & Sub15 & Sub17 & Sub19 \\
$\mathrm{n}(\%)$ & $\mathrm{n}(\%)$ & $\mathrm{n}(\%)$ & $\mathrm{n}(\%)$ \\
\hline Ótima & $8(88,88)$ & $9(69,23)$ & $2(28,57)$ & $5(55,55)$ \\
Normal & $1(11,11)$ & $2(15,38)$ & $3(42,85)$ & $2(22,22)$ \\
Limítrofe & -- & $2(15,38)$ & $0(00,00)$ & $2(22,22)$ \\
Hipert Sist Isolada & -- & -- & $2(28,57)$ & -- \\
\hline
\end{tabular}

\section{DISCUSSÃO}

A discussão do estudo será feita com modalidades que são similares ou parecidas com o basquetebol, quanto ao gasto e à utilização das demandas energéticas, às capacidades condicionantes, à resistência aeróbica e anaeróbica, à velocidade e à força, uma vez que são raros os estudos experimentais com atletas de basquetebol.

O estudo de Furtanetto ${ }^{14}$, realizado com 160 atletas que disputaram as quartas de final do campeonato de futebol amador de Uberlândia, com objetivo de analisar a prevalência de fatores de risco cardiovasculares, concluiu que houve pequena prevalência do fator obesidade nos atletas. Esses resultados vêm corroborar com a presente pesquisa em que a maioria dos atletas analisados também apresentou baixa prevalência de obesidade.

O trabalho de Campos ${ }^{15}$, feito com 160 atletas que disputaram o campeonato de handebol, com o objetivo de verificar a prevalência de fatores de risco, ressaltou que a PA sistólica e a PA diastólica de repouso encontraram-se dentro na normalidade em ambos os sexos. Dados esses que corroboram com o presente estudo em que a maioria dos avaliados quanto à Pressão Arterial mostrouse na classificação normal.

Já no estudo de Oliveira, Rodrigues ${ }^{16}$, constando 76 atletas de futebol de campo dividido em dois grupos: 38 atletas profissionais e 38 atletas sub-20, teve como o objetivo comparar o perfil antropométrico: peso, altura e IMC. Nesse trabalho as variáveis antropométricas (peso, altura e IMC) não apresentaram diferenças estatisticamente significativas entre os grupos. Mais uma vez esses resultados podem ser vistos no presente estudo, uma vez que, ao comparar as quatro categorias, não houve diferença expressiva entre as categorias de sub13 a Sub19.

O estudo de Dias ${ }^{17}$, feito com 32 atletas jogadores de voleibol da Associação Esportiva e Recreativa Usip, com o objetivo de identificar e comparar os valores antropométricos, Peso, Estatura, IMC e percentual de gordura, obteve como resultado da amostra avaliada valores normais. Entretanto foi de encontro ao presente estudo, pois mostrou que, de nove atletas da categoria sub19, oito deles apresentaram valores que se enquadram como sobrepeso. 
A pesquisa de Paiva ${ }^{18}$ foi o único estudo encontrado com participantes de basquetebol, foi realizado com 85 atletas jogadores da Liga Nacional, tendo como objetivo avaliar a composição corporal de mais de um terço dos atletas da elite do Brasil.

\section{Conclusão}

Esse estudo concluiu que os índices de percentual de gordura e IMC da maioria dos atletas encontrava-se dentro do recomendado para a saúde. Dados estes que contribuem com o estudo atual, uma vez que a maioria dos participantes se encontraram na classificação normal. Chegou-se ao resultado de que a maioria dos atletas desse estudo não apresenta riscos cardiovasculares..

Ao correlacionar as variáveis, constatou-se que, à medida que os atletas vão ficando mais pesados e mais altos, maior é a Circunferência de Cintura e o Índice de Massa Corporal. E quanto comparado as categorias constatou que a categoria sub19 obteve maiores valores em todas as variáveis: Peso, Estatura, CC, IMC e PAS, com exceção da variável PAD.

\section{REFERÊNCIAS BIBLIOGRÁFICAS}

1. Silva JVF, Silva EC, Silva EG, Ferreira AL, Rodrigues APRA. Perfil da morbidade hospitalar por doenças respiratórias na infância de 0 a 9 anos na cidade de Maceió - AL no período de 2010 a 2014. Ciênc. Biol. Saúde. 2016;3(3):43-58.

2. Nascimento BR, Brant LCC, Oliveira GMM, Malachias MVB, Reis GMA, Teixeira RA, et al. Epidemiologia das Doenças Cardiovasculares em Países de Língua Portuguesa: Dados do \&quot; Global Burden of Disease \& quot; 1990 a 2016. Arq. Bras. Cardiol.2018;110(6):500-11. doi:10.5935/abc.20180098.

3. Kiuchi MG, Mion Junior D. Doença renal crônica e fatores de risco para morte súbita cardíaca - denervação renal simpática: um sopro de esperança? Relampa. 2016;29(3):108-19.

4. Filgueira FW, Silva FG, Soares TCM, Gomes JGN. Nível de aptidão física em praticantes das modalidades esportivas da escola municipal Maria de Lourdes Cavalcante. Red Foco. 2016;3(1):27-45.

5. Bittencourt MI, Cader AS, Araújo DV, Salles ALF, Albuquerque ALFN, Spineti PPM, et al. Papel da fibrose miocárdica na cardiomiopatia hipertrófica: revisão sistemática e metanálise atualizada de marcadores de risco para morte súbita. Arq. Bras. Cardiol. 2019;112(3):281-89. doi:10.5935/abc.20190045.

6. Folle A, Collet C, Salles WN, Nascimento JV. Transições no processo de desenvolvimento de atletas do basquetebol feminino. Rev. Bras. Educ. Fís. Esporte. 2016;30(2):477-90. doi: 10.1590/1807-55092016000200477.

7. Menegassi DA, Sabbag AA, Costa ARC, Maltauro L, Melo TR, Neves EB. Terapia neuromotora intensiva melhora a composição corporal na paralisia cerebral. RBONE. 2019;13(78):275-83.

8. Caritá RAC, Pessoa Filho DM, Barbosa LF, Greco CC. Componente lento da cinética do vo2: determinantes fisiológicos e implicações para o desempenho em exercícios aeróbios. Rev. Bras. Cineantropom. Desempenho Hum. 2014 ;16(2): 233-246. doi: 10.5007/19800037.2014v16n2p233.

9. Lima LRA. Perspectivas atuais em atividade física e saúde de crianças e adolescentes que vivem com HIV. Rev. Biom. 2018;12(1):1-7.

10. Vargas AJ, Pessoa LS, Rosa RL. Jejum intermitente e dieta Low Carb na composição corporal e no comportamento alimentar de mulheres praticantes de atividade física. Rev. Bras. Nutr. Espor. 2018;12(72):483-90.

11. Santos IA, Passos MAS, Cintra IP, Fisberg M, Ferreti RL, Ganen AP. Pontos de corte de circunferência da cintura de acordo com o estadiamento puberal para identificar sobrepeso em adolescentes. Rev. Paul. Pediatr. 2019;37(1):49-57. doi: 10.1590/1984-0462/;2019;37;1;00003. 
12. Galatti LR, Paes RR, Machado GV, Gonçalves CEB, Seoane AM. Determinantes de excelência no basquetebol feminino: as conquistas da seleção brasileira na perspectiva das atletas. Rev. educ. fis. 2015;26(4):621-32. doi: 10.4025/reveducfis.v26i4.26424.

13. Bittencourt MI, Cader S, Araújo DV, Salles ALF, Albuquerque FN, Spineti PPM et al. Papel da fibrose miocárdica na cardiomiopatia hipertrófica: revisão sistemática e metanálise atualizada de marcadores de risco para morte súbita. Arq. Bras. Cardiol. 2019;96(3):193-196. doi: 10.5935/abc.20190045.

14. Furtanetto Júnior R, Gonçalves A. Prevalência de fatores de riscos cardiovasculares em atletas de futebol amador da cidade de Uberlândia/MG. Rev. Dig. 2008;13(119):1-9.

15. Campos AC. Prevalência de fatores de risco cardiovascular em atletas de handebol participantes do Jimi. [Monografia]. Uberlândia: Centro Universitário do Triângulo; 2005.

16. Oliveira LF, Rodrigues PAS. Circunferência de cintura: protocolos de mensuração e sua aplicabilidade prática. Rev. Nutr. Vigilân. Saúde. 2016;3(2):90-5.

17. Dias PC, Henrique P, Anjos LA, Burlandy L. Obesidade e políticas públicas: concepções e estratégias adotadas pelo governo brasileiro. Cad. Saúde Pública. 2017;33(7):17-26. doi: 10.1590/0102-311x00006016

18. Paiva Neto A, César MC. Avaliação da composição corporal de atleta de basquetebol do sexo masculino participantes da liga nacional 2003. Rev. Bras. Cineantrop. 2005;7(1):35-44. 